\title{
Review Article \\ Fundamental Issues and Prospective Directions in Networked Multirate Control Systems
}

\author{
Wang Zhiwen ${ }^{1,2}$ and Guo Ge ${ }^{2}$ \\ ${ }^{1}$ College of Electrical and Information Engineering, Lanzhou University of Technology, Lanzhou 730050, China \\ ${ }^{2}$ School of Control Science and Engineering, Dalian University of Technology, Dalian 116026, China \\ Correspondence should be addressed to Wang Zhiwen; wwwangzhiwen@163.com
}

Received 4 December 2013; Revised 15 February 2014; Accepted 16 February 2014; Published 19 March 2014

Academic Editor: Hak-Keung Lam

Copyright (c) 2014 W. Zhiwen and G. Ge. This is an open access article distributed under the Creative Commons Attribution License, which permits unrestricted use, distribution, and reproduction in any medium, provided the original work is properly cited.

\begin{abstract}
In view of the synchronized uniform equidistant sampling method that is widely used in network control systems (NCSs), multirate sampling control for NCSs is presented by considering the natural characteristics of NCSs. Two kinds of typical structures of network multirate control systems (NMCSs) are presented and the multirate sampling mechanisms are formulated. Then, fundamental issues and prospective directions in NMCSs are preliminarily discussed. The contents involve modeling, sampling pattern selection, control strategies, scheduling algorithms, and codesign of NMCSs. The purpose of this paper is to arouse more attention of researchers in related subjects, so that a complete theory for NCSs can be established.
\end{abstract}

\section{Introduction}

In the past decade, we were faced with the significant advances of computer science and communication technology. The unprecedented interactions and penetrations between developments in computer, communication, and control have accelerated the progress of the networked control. Motivations for using the networked framework in control systems come from lower cost, ease of maintenance, great flexibility, and sharing of information resources, which make networked control systems (NCSs) more and more popular. NCSs are comprised of the plant to be controlled and some components (sensors, controller, actuators, etc.) whose operation is coordinated through a real-time communication network. The nature characteristic of NCSs is that information, such as reference input, plant output, and control signals, is exchanged among control system by communication network and multisubsystem sharing this information to accomplish their respective control tasks. These features make NCSs as the preferred architecture in modern largescale complex system. Examples comprise spatially distributed resource allocation networks, supervisory control of continuous plants, intelligent vehicle highway systems, power generation and distribution networks, mobile sensor networks, remote surgery, and many others. Consequently, considerable attention has been directed to the study of NCSs; see, for example, the survey papers [1-3], the recent special issues $[4,5]$, and the references therein.

NCSs are typical multivariable and multiloop computer control systems with both continuous-time analog and discrete-time digital signals inside. In the course of system modeling, analysis, and design, the problems of signal sampling and holding will be involved inevitably. The existing achievements of NCSs were almost employing the fundamental assumption about sampler and holder of traditional control theory; that is, the sampling rate of each node in NCSs is identical and synchronous. Such uniformly spaced samples may result in easier reconstruction algorithms and bring more convenience for the theoretical research of NCSs. However, in view of the essential features such as largescale, complicated framework and high distribution level, it is unrealistic for NCSs to acquire all nodes' information by adopting the traditional sampling manner in practical. Therefore, the previous theoretical achievements based on synchronous uniformly spaced sampling are questionable and should be reconsidered. 
Multirate sampling control is motivated by practical implementation of aerospace system originally [6] and is of prominent interest in current control research. Further utility of multirate sampling in control applications can be found for highly distributed systems, such as communication networks and power generation and distribution networks. These examples can be regarded to some extent as the preliminary applications of multirate sampling in NCSs. But, to the best of the author's knowledge, lesser attention has been directed to multirate sampling theory of NCSs and few literatures address the related issues deeply till now. In [7], modeling problem of NCSs is discussed by taking not only network-induced time delay, but also asynchronous multirate sampling into account. Optimal multirate control of the NCSs is investigated in [8]. By using lifting technology, linear periodic time-invariant model of NCSs with multirate sampling is established. Then, controllability and observability are analyzed and optimal state and output feedback control are given. In [9], model-based control scheme of NCSs with multirate input sampling is studied. State feedback and output feedback control are discussed, respectively. Necessary and sufficient conditions for system to be globally exponentially stable are derived. In [10], the stabilization of multirate networked control systems is investigated. The key idea is to use the channel resource allocation to stabilize the system. Conclusions show that a multirate networked control system could be stabilized by state feedback under an appropriate resource allocation. The result can also be applied to multirate quantized networked control systems. In [11], model predictive control (MPC) for NMCSs with the output sampling period several times larger than the input updating period is investigated. Sufficient stability conditions are established via a switched Lyapunov function approach and a controller design method for stabilising NMCSs is proposed. In [12], a multirate method was proposed to formulate NCSs with network-induced time delays as jump linear systems and the sufficient conditions of stability were established. In [13], the NCSs problem was formulated as a dual-rate control problem: low transmission rate for data over the network and high updating rate for actuator commands. In [14], an adaptive sampling rate scheduling methodology is proposed to maintain the transmitted measurement signals' fidelity and conserve available bandwidth in order to help supervisory control system under normal and abnormal operating conditions. As a complement, adaptive multiple sampling rate scheduling algorithm is presented in [15] to dynamically adjust sampling rates and allocate available bandwidths to transmit measurements from the remote plants to the supervisors.

The above-mentioned works can be divided into two categories. One mainly discusses the basic problems of NCSs with multirate sampling, such as modeling, controllability and observability, stabilization and stability analysis. The other focuses on the problems of scheduling scheme, data rate control, and bandwidth allocation, which are closely related to system sampling period and expected to achieve by means of variable sampling or multirate sampling. These works are worthy of recognition for their contributions; meanwhile, we have to realize that great efforts must be made for further

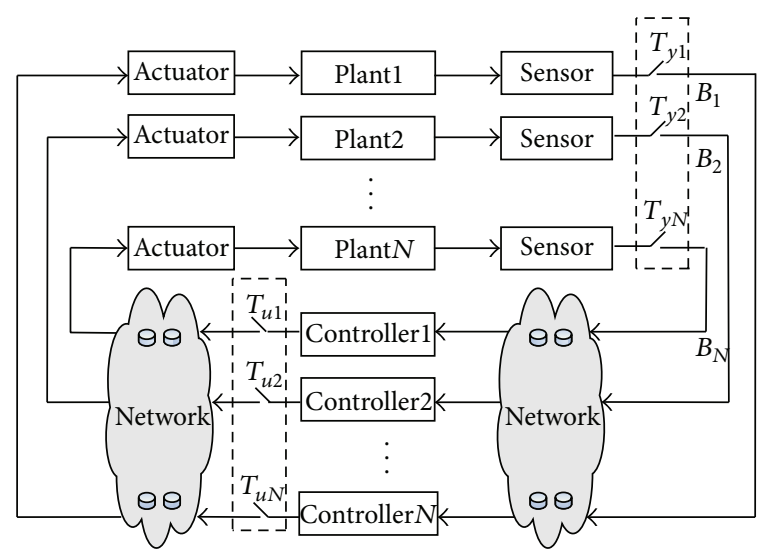

$\Theta$ Data packet

FIGURE 1: GM-NCSs in the direct structure.

pursuit and understanding of the basic theory of NCSs with multirate sampling. Inspired by this, this paper discusses the fundamental issues of networked multirate control systems (NMCSs) tentatively.

The remainder of this paper is organized as follows. In Section 2, two kinds of typical structures of NMCSs are presented and the mechanisms of multirate sampling are described. In Section 3, fundamental issues and prospective directions are discussed tentatively in time domain. Section 4 summarizes the paper and puts forward the future works.

\section{System Descriptions}

There are two general NCSs configurations termed as direct structure and hierarchical structure [1]. The direct structure is the most common NCSs structure where the controller and the plant are physically located at different locations and are directly linked by a data network in order to perform remote closed-loop control as illustrated in Figure 1. The hierarchical structure NCSs is also called networked supervisory control system. It consists of a main controller and remote closedloop subsystems as depicted in Figure 2. The main controller computes and sends the reference signal in a frame or a packet via a network to the remote system. The remote system then processes the reference signal to perform local closed-loop control and returns to the sensor measurement to the main controller for networked closed-loop control.

Generally speaking, according to the relationship between each sampling period, multirate sampling system can be divided into the following three categories: multirate input control system (MRICS), multirate output control system (MROCS), and generalized multirate control system (GMCS). MRICS and MROCS can be seen as the special case of GMCS. In MRICS, the sampling rate of the input channel is higher than that of output channel. Namely, in a certain time interval, one time samples the output and several times the input. Therefore, MRICS is also known as fastslow sampling system. On the contrary, the sampling rate of the output channel is higher than that of input channel in 


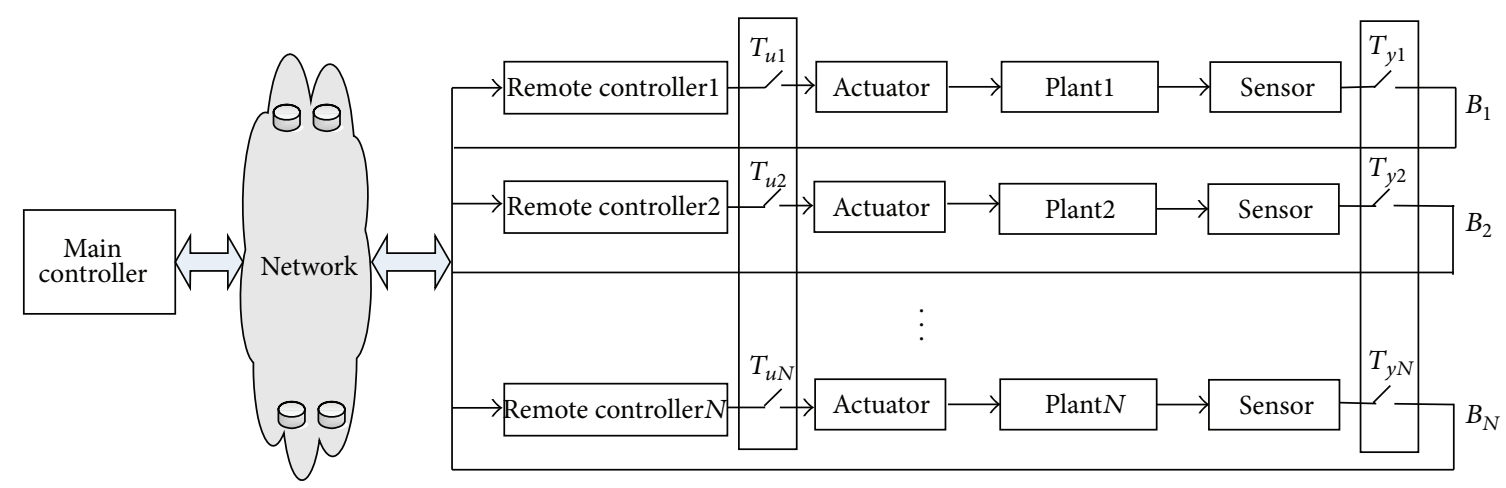

Data packet

FIGURE 2: GM-NCSs in the hierarchical structure.

MROCS, and it is referred to as slow-fast sampling system. Based on the above principle, we can classify NMCSs as MRINCSs, MRO-NCSs, and GM-NCSs. Figures 1 and 2 show two typical structures of GM-NCSs.

Consider the GM-NCSs consisting of a collection of continuous time LTI plants, as illustrated in Figures 1 and 2, where the $i$ th plant is given by

$$
\begin{gathered}
x_{i}(t)=A_{i} x_{i}(t)+B_{i} u_{i}(t), \\
y_{i}(t)=C_{i} x_{i}(t),
\end{gathered}
$$

where $x_{i} \in \mathfrak{R}^{n_{i}}, u_{i} \in \mathfrak{R}^{m_{i}}$, and $y_{i} \in \mathfrak{R}^{p_{i}}$ are the system state, the control input, and the output, respectively, and $i=$ $1,2, \ldots, N$ denotes the loop number. $A_{i}, B_{i}$ and $C_{i}$ are the real matrices with appropriate dimensions.

For convenience of investigation, reasonable assumptions are made that all sensors, controllers, and actuators are driven by synchronized clock. Then, the multirate sampling process can be summarized as follows. In MRI-NCSs, the plant outputs are sampled with the same sampling period $T_{0}$ and the plant input of different loop is sampled with different period $T_{u i}$, where $T_{0}$ is the cyclic period of the whole system and known as the frame period. Namely, in a frame period $T_{0}$, one time samples the output and several times the input. Similarly, in MRO-NCSs, the plant inputs are sampled with the same sampling period $T_{0}$ and the plant output of different loop is sampled with different period $T_{y i}$. In GM-NCSs, the inputs and outputs are sampled with $T_{u i}$ and $T_{y i}$ in a frame period, respectively.

\section{Fundamental Issues and Prospective Directions of NMCSs}

The research of multirate sampling has experienced two stages: frequency domain and time domain. Time-domain method was initiated by Kalman and Bertram [16] in 1959 and is the dominating method for its power and flexibility in characterizing many types of sampled data control systems till now. To further understand the interaction between control and communication in NMCSs, fundamental issues, such as modeling and controller design, will be discussed tentatively
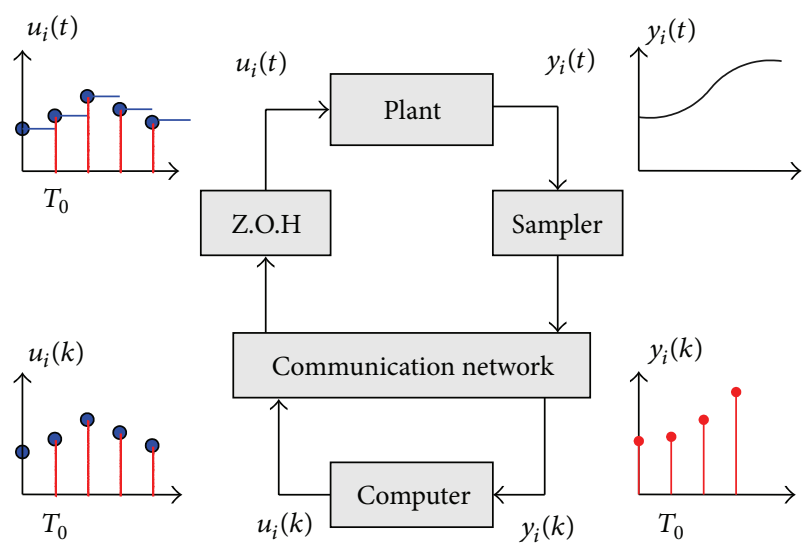

FIGURE 3: Schematic diagram of single-rate sampled-data system.

in time domain in the following, and it is an important initial step toward some larger goals.

3.1. Modeling of NMCSs. In conventional single-rate sampled-data systems, the controller switch at the sampling instant and the control action is held at the same time, as shown in Figure 3. Sampling (1) with period $T_{0}$, the discrete-time model of the whole system can be described by

$$
\begin{gathered}
x_{i}(k+1)=\Phi_{i} x_{i}(k)+\Gamma_{i} u_{i}(k), \\
y_{i}(k)=C_{i} x_{i}(k),
\end{gathered}
$$

where $\Phi_{i}=e^{A_{i} T_{0}}, \Gamma_{i}=\int_{0}^{T_{0}} e^{A_{i} t} B_{i} d t$ and $k \in \mathrm{Z}_{0}^{+}$(nonnegative integers) is the time index.

Different from single-rate sampled-data systems, modeling of NMCSs is more complicated since both the inherent characteristics of communication network and the particularity of multirate sampling will impact the mathematical model dramatically.

One main issue in communication network is the presence of network-induced delays stemming from the fact of utilizing a common communication channel for closing the loop as well as additional functionality required for physical signal coding and communication processing. According 
to the direction of data transfers, network-induced delay can be categorized as the sensor-to-controller delay $\tau^{\text {sc }}$ and controller-to-actuator delay $\tau^{\mathrm{ca}}$. It is clear that networkinduced delays result in the disorder and missynchronization of the sampled sequence inevitably and may introduce vacant sampling and message rejection, as shown in Figure 4.

The occurrence of network-induced delay not only impacts the dynamics of both plant and controller but also degrades control performance and introduces potential instability. In [17], the effects of the network-induced delays, that is, data latency, message rejection, and vacant sampling, on the system dynamic performance are systematically considered. In [18], discrete-time modeling approaches for four significant classes of NCSs were presented. In [19], modeling and control of NCSs with different loop topology structures are considered and the uniform discrete state-space model is established by introducing state augmentation for both shortand long-time delays. In summary, modeling of NCSs with network-induced delay has received considerable attentions and some preferable results were obtained.

Modeling of multirate sampling system is always a difficult problem since the distinction of sampling periods between input and output channel and the missynchronization of the sampled sequence. For the sake of simplicity, assumptions are always made that the sampling period of input channel is operated at a rate some integer ratio higher or lower than that of the output channel. Figure 5 shows the schematic diagram of MRI-NCSs with $T_{0}=2 T_{u i}$ and MRONCSs with $T_{0}=3 T_{y i}$, respectively.

For example, in MRI-NCSs, the relationship between $T_{0}$ and $T_{u i}$ fulfills $T_{0}=N_{i} T_{u i}$, where $N_{i} \in \mathrm{Z}^{+}$(positive integers). Let $N_{0}=\operatorname{LCM}\left(N_{1}, \ldots, N_{N}\right)$, where $\operatorname{LCM}(\cdot)$ is the least common multiple. The basic sampling period of MRI-NCSs can be defined as $T=T_{o} / N_{0}$. In view of the characteristic of the multirate input sampling, using the "lifting" technique and defining the augmented input vectors as follows:

$$
u_{e}\left(k T_{0}\right)=\left[\begin{array}{c}
u_{e 1}\left(k T_{0}\right) \\
u_{e 2}\left(k T_{0}\right) \\
\vdots \\
u_{e N}\left(k T_{0}\right)
\end{array}\right]
$$

where

$$
u_{e i}\left(k T_{0}\right)=\left[\begin{array}{c}
u_{i}\left(k T_{0}\right) \\
u_{i}\left(k T_{0}+T_{u i}\right) \\
\vdots \\
u_{i}\left(k T_{0}+\left(N_{i}-1\right) T_{u i}\right)
\end{array}\right], \quad i=1,2, \ldots, N .
$$

Then, for periodic sampling with constant period $T_{0}$, the linear time invariant state space model of the whole system can be established [20].

Remark 1. It can be seen from Figures 4 and 5, to eliminate vacant sampling and message rejection in NMCSs, the following conditions must be held: (1) $\tau<T_{u i}$ for MRI-NCSs; (2) $\tau<T_{y i}$ for MRO-NCSs; (3) $\tau<\min \left(T_{u i}, T_{y i}\right)$ for GM-NCSs, where $\tau=\left\{\tau^{\mathrm{sc}}, \tau^{\mathrm{ca}}\right\}$.

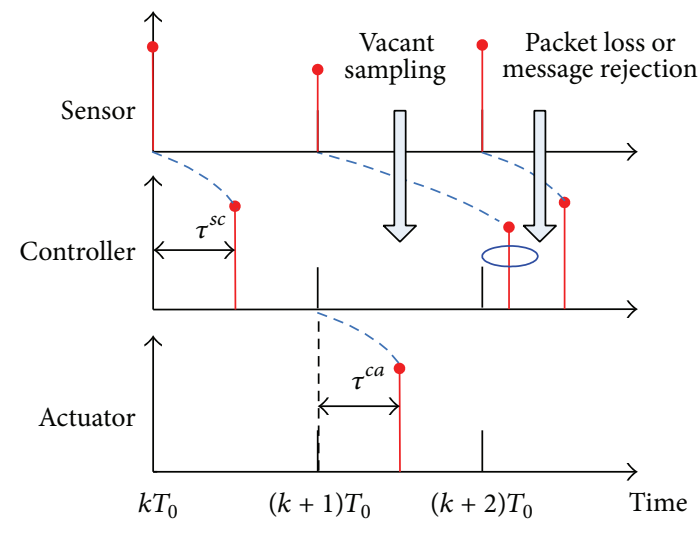

FIgURE 4: Timing diagram of NCSs.

From the above analysis we can draw a conclusion that both the adverse factors caused by network-induced delay and the missynchronization of the sampled sequence introduced by multirate sampling pattern should be taken into account in the course of NMCSs modeling. But, to the author's knowledge, few literatures considered these issues comprehensively. In [7], under the assumption of delay bounded by sampling period, modeling problem of NCSs is discussed by introducing the definition of discrete lifting. Modeling of MRO-NCSs and MRI-NCSs are established, respectively, but no hints on GM-NCSs and the case of long-time delay. In [21], the models of multirate networked control systems with no network-induced delay and longtime delay are proposed, respectively. It is stated that the multirate networked control systems with long-time delay can be formulated as a discrete-time switched stochastic system.

Remark 2. In the previous, we have made the assumption that all sensors, controllers, and actuators in NMCSs are driven by synchronized clock. That is to say, all these components work with their own period. Time-driven manner can avoid the disorder of the sampled sequence on the one hand but will introduce additional time delay, which degrades control performance, on the other hand.

Remark 3. Network-induced delay is originated in the very fact of utilizing the shared communication network with limited capacity and data rate. It is impracticable to eliminate network-induced delay since it is the intrinsic property of communication network. So, the emphasis should be put on the minimization of the delay rather than elimination. Fortunately, this objective is expected to achieve by means of multirate sampling and/or variable sampling which will be discussed in what follows.

Remark 4. The introduction of the augmented vectors leads to the high dimensionality of the system model and makes the analysis and design of the system complicated. So, the reduction of unnecessary augmented vectors and dimensions of the state space model are key issues worthy of considering. 


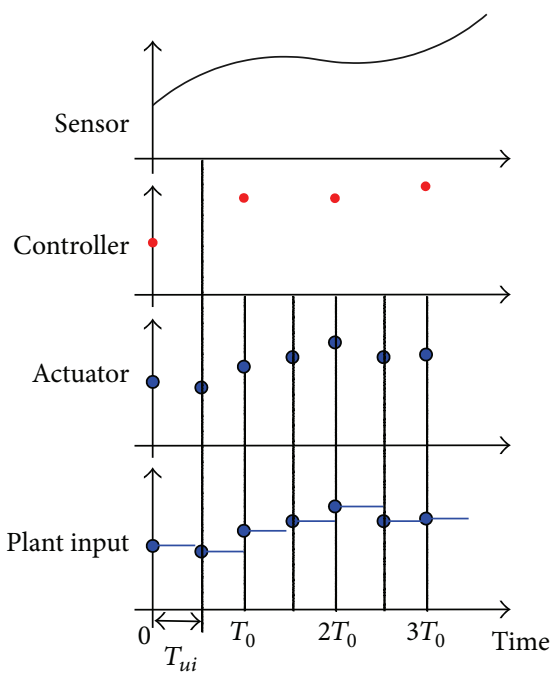

(a) MRI-NCSs $\left(T_{0}=2 T_{u i}\right)$

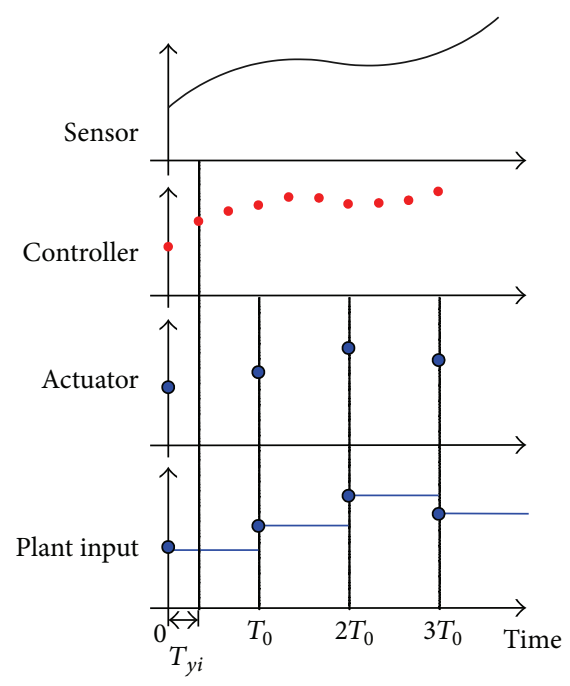

(b) MRO-NCSs $\left(T_{0}=3 T_{y i}\right)$

FIGURE 5: Schematic diagram of multirate sampling system.

3.2. Sampling Guaranteeing Controllability and Observability. Controllability and observability are of the two basic concepts to describe the internal structure characteristics of the control systems. They appear as necessary and sometimes as sufficient conditions for the existence of a solution to most control problems and play a central role in modern control theory.

In NMCSs, the plant inputs (and/or outputs) are sampled with different sampling periods in one frame period. Compared with equidistant periodic sampling pattern, the acceleration of sampling frequency in one frame period in multirate sampling can acquire more information about the system and will improve the controllability and observability to some extent. But, on the other hand, when a continuoustime system is converted into discrete-time form by means of a sample and hold operation, the improper selection of the sampling periods will cause the change of the system controllability and observability.

In a general way, a condition on the sampling period $T$, which is sufficient (and in many cases also necessary) to rule out pathological situations, is that

$$
T \neq \frac{2 l \pi}{\operatorname{Im}\left(\lambda_{i}-\lambda_{j}\right)}, \quad l= \pm 1, \pm 2, \ldots,
$$

where $\lambda_{i}, \lambda_{j}$ are of different eigenvalues of the continuoustime system. Based on the above condition, controllability and observability of multirate sampling control system are discussed and the conditions for system to be controllable and observable are presented in [20]. A nonequidistant periodic sampling pattern, which has the property to guarantee the controllability and observability during discretization, is proposed in [22] and is further discussed in [23], but no hints on the selection of the sampling rates. To the author's knowledge, besides some empirical formulae and conventions, there is hardly any literature discussing the topic of sampling period selection in multirate control system. These backgrounds strongly motivate the interest in pursuing for the sampling patterns and the selection of sampling rate in NMCSs.

3.3. Multirate Sampling Control Strategies of NCSs. The introduction of multirate sampling in NCSs makes the analysis and design of the system more complex. Meanwhile, it is possible for NCSs to achieve some unexpected control objectives since the periodic time-varying controller of multirate sampling can increase the degrees of freedom in controller design and improve the control performance to some extent. In [24], pole placement for multirate sampled linear systems by output feedback is studied. The presented approach allows for simultaneous selection of the sampling regime and the closed-loop poles. Also, pole-placement problem is solved by resorting to a controller composed by a periodic state observer and a nondynamic control law in [25]. In [26, 27], optimal control for multirate systems is discussed and stability robustness issues in periodic and multirate systems are analyzed. In [28], the strong and simultaneous stabilization problems for discretetime linear time-invariant systems are completely solved by periodic time-varying controllers. In [29], a decentralized control approach to multirate sampled-data systems bared on lifting technique is considered and a reliable decentralized multirate controller design is presented. Robust performance and robust stability of the decentralized multirate systems can be achieved by using the design method proposed. In [30], the authors study the use of generalized sampled-data hold functions (GSHF) in the problem of simultaneous controller design for linear time-invariant plants. In [31], solutions to the simultaneous regulation problem of multirate systems are presented. In [32], the problem of pole assignment of decentralized multirate sampling system is discussed. It can be seen that the periodic time-varying controllers of multirate system may achieve some special functions which cannot 
be realized by conventional linear time-invariant controller. Some of these functions, such as robust control, simultaneous stabilization, and decentralized control, are the desired control objectives in NCSs design.

In MRICS, the sampling rate of the input channel is higher than that of output channel. The acceleration of the input sampling rate is equivalent to the increase of the effective input. From the point of view of the continuous time, feedback control is performed in both the sample instant and the intersample, which will strengthen the control ability to the plant of the controller. In MROCS, the plant outputs are measured several times in a frame period and more information about the plant is obtained. The extension of effective output of the system corresponds to the increase of the controller's input, which will strengthen the control ability of the controller, especially the output feedback controller. So, how to make full use of the above characteristics of MRI and MRO to design the periodic time-varying control strategies is a major concern and worthy of further considering.

\subsection{Scheduling of NCSs Based on Multirate Sampling and} Variable Sampling. NCSs include control and network; the performance of control loops depends not only on the design of the control strategies but also on the scheduling of the shared network resources. Sampling period plays an important role between control system and communication network in NCSs. A larger sampling period means lower data transmission rate, which can help to reduce the networkinduced time delay and information conflict in a communication network. However, it will lead to the sacrifice of control performance and the reduction of the network utilization, which affects the overall performance of NCSs. On the contrary, smaller sampling period makes the best use of the network utilization and will improve the control performance consequently but aggravates the network congestion and may result in delays and packet dropouts, which will also affect NCSs performance.

Constant sampling is the simplest and most traditional sampling methods in NCSs. It refers to the sampling period in the process of system operation remains the same. It is worth noting that, if constant sampling period is adopted, it should be large enough to avoid network congestion when the network is occupied by the most users. Clearly, the constant sample period results in network resource waste and may obtain the conservative results for the NCSs. Motivations for using variable sampling and multirate sampling in NCSs come from the full use of the limited network resource and the reduction of network-induced delays and its effects on system.

RM (rate monotonic) and EDF (earliest deadline first) [33] are traditional real-time scheduling algorithms and theoretically applicable to control network scheduling. RM algorithm is a kind of statistic scheduling methods. The priority of each task is determined before being executed and not changed along with time. Moreover, RM algorithm is a preemptive algorithm; that is, the currently executing task is preempted by a newly arrived task with shorter period. In [34], an improved RM algorithm is put forward and sufficient condition for the network to be schedulable is given. EDF algorithm is a kind of dynamic scheduling algorithms. The priority of each task is determined according to the time vary to the deadline of each task; the smaller the time varying, the higher the task priority. Task priority is not fixed but changes along with the time. RM and EDF algorithms are all "open loop" scheduling algorithms. Once the scheduling mechanisms are created, they cannot adjust themselves with the changing of the system. They can perform well in predictable environments in which the network loads can be accurately modeled but poorly in unpredictable environments.

In view of the disadvantages of traditional scheduling algorithms, scheduling algorithms based on the adjustment of sampling period dynamically are emerged. In [35], the influence of sampling period on the performance of NCSs is analyzed systematically and the guidelines for obtaining the optimal working range of sampling times are provided. By analyzing the influence of sampling period, the performance of control systems is studied in [36,37]. In [38], a scheduling algorithm which could allocate the bandwidth and determine the sampling periods of sensors under constraint that the networked-induced delays were less than the sampling periods is presented. Concerned with different types of data, a scheduling method for NCSs was proposed in [39] under the assumption that the other control loops' sampling period was the integral multiples of the basic sampling period. In [40], a time window algorithm to determine the sampling period to reduce the effect of delay is proposed. In addition, a calculational method to show the relationship between networkedinduce delay and the sampling period was proposed in [41], but how to optimize the sampling period is not considered. In [42-44], multiloop sampling period optimization problems are analyzed with nonlinear programming and Kuhn-Tucker theory. In [45], an adaptive sampling rate scheduling method is proposed to allocate the limited network resource based on the requirement of QoP and QoS. The closed-loop system is modeled as a discrete switched system, and then the stability condition and switched feedback controller design method are given based on switched theory.

In our previous works [46-48], variable sampling and multirate sampling scheduling algorithms are put forward by online supervision of the control performance and the available bandwidth. We considered the networked DC-Motor with transfer function $G(s)=1000 /\left(s^{2}+s\right)$. The parameters of $P-D$ control algorithm can be described by

$$
\begin{gathered}
P(k)=K(r(k)-y(k)), \\
D(k)=a_{d} D(k-1)+b_{d}(y(k-1)-y(k)), \\
u(k)=P(k)+D(k),
\end{gathered}
$$

where $a_{d}=T_{d} /\left(N h+T_{d}\right), b_{d}=N K T_{d} /\left(N h+T_{d}\right), N=100000$, $T_{d}=0.05, K=0.6$, and $h=0.0075$. The performance of the proposed algorithms is compared with RM and EDF scheduling algorithms in TrueTime toolbox under Matlab environment, as shown in Figure 6. It is clear that the variable sampling algorithm shows a good performance in overshoot, stability, and the suppression on the influence of time delay than that of RM and EDF algorithm. 


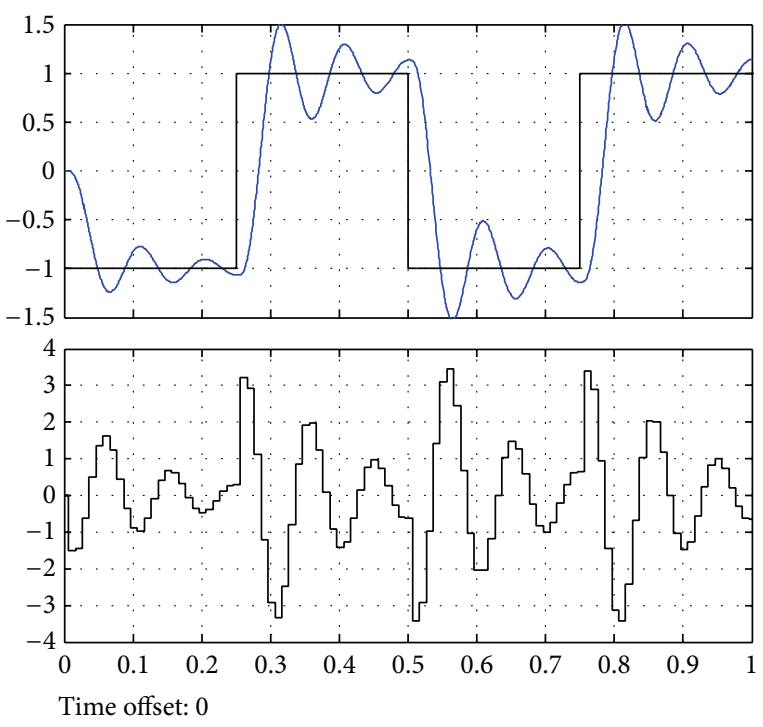

(a) RM algorithm

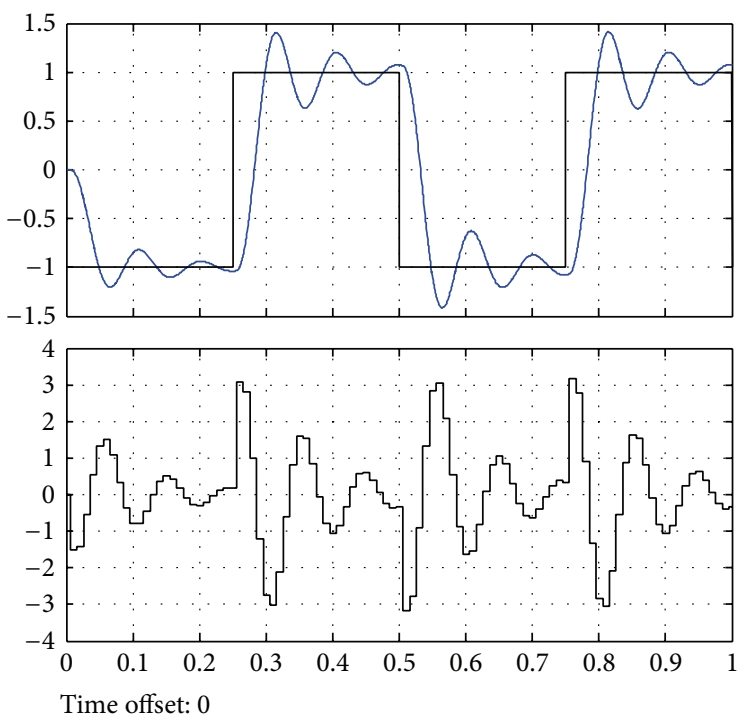

(b) EDF algorithm
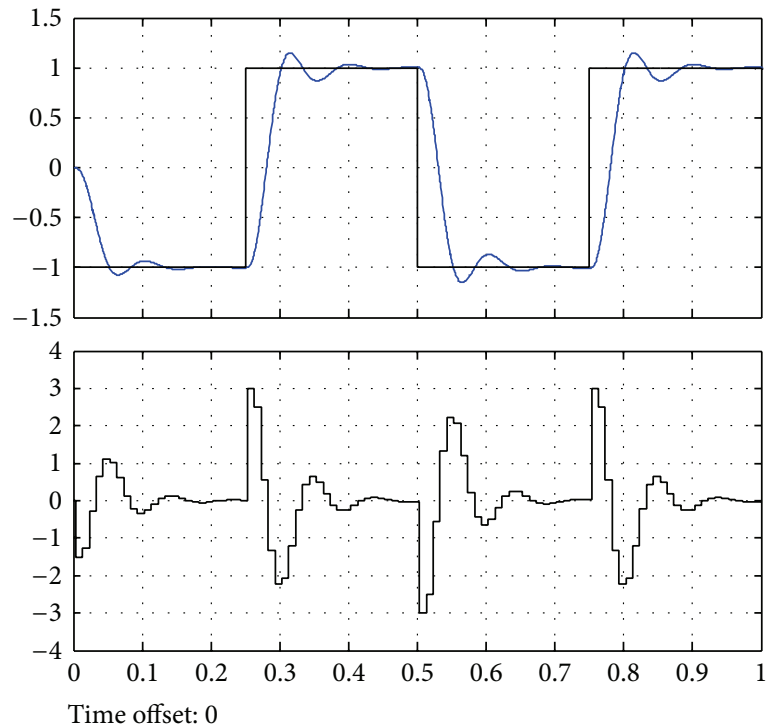

(c) Variable sampling algorithm

FIGURE 6: Control performance of different scheduling algorithms.

3.5. Codesign of NMCS. There have reached a consensus that the whole performance of NCSs correlated with not only the control algorithms but also the network resource allocation. Namely, a successful NCSs design should consider the quality of performance (QoP) of the control system and the quality of service (QoS) of the communication network simultaneously. Hence, the thought of control and scheduling codesign is put forward in [49] in order to resolve the problems caused by QoP and QoS which influence and restrict each other mutually.

Denote $B_{g}$ as the available global bandwidth of communication network and $B_{i}$ as the necessary bandwidth for loop $i$ to accomplish its control task, as shown in Figures 1 and 2. With traditional constant sampling manner in NCSs, all subsystems will transfer data synchronously and occupy the bandwidth averagely. That is, $B_{1}=B_{2}=\cdots=B_{N}$ and $\sum_{i=1}^{N} B_{i}=B_{g}$. Such bandwidth allocation mechanism is obviously the most simple manner and ease of realizing, on one hand, but, on the other hand, it will cause the waste of network bandwidth resources, the aggravation of networkinduced delay, and the decline in overall quality of the system, even worse.

Multirate sampling, considering either the physical properties of the controlled plants or the limitations of the communication network, is an inevitable choice of NCSs. In NMCSs, different control loop is sampled with different sampling period, which can realize the reasonable allocation of the bandwidth resources and guarantee the QoS of the communication network. The distribution principle is that the higher the sampling rate is, the bigger the bandwidth would 


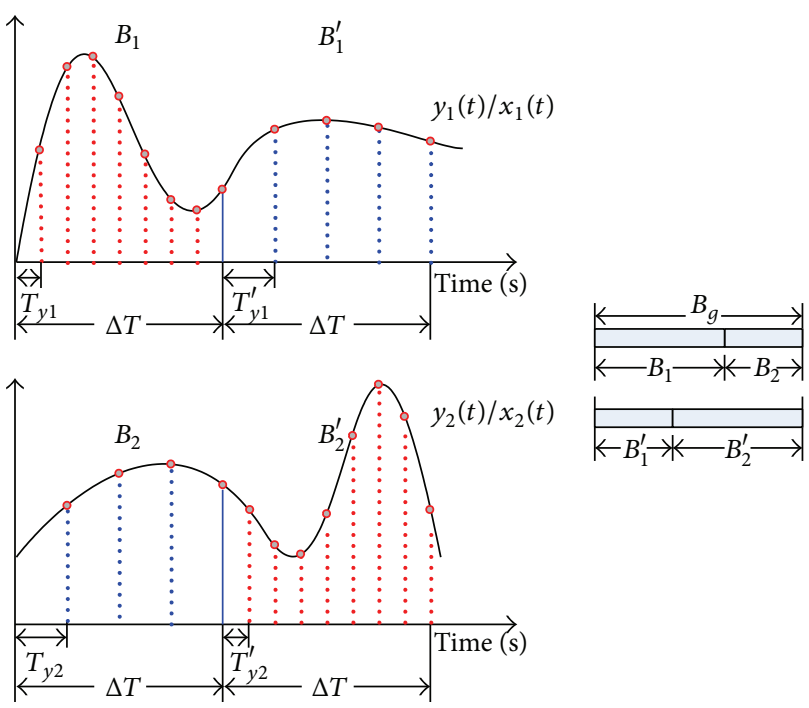

FIGURE 7: Schematic diagram of dynamic bandwidth allocation.

be. For example, in MRO-NCSs, suppose that the sampling periods of the $N$ output channels are $T_{y 1} T_{y 2} \cdots T_{y N}$ and $T_{y i} \leq T_{y(i+1)}$. Then, the bandwidth assigned to the $i$ th subsystem can be calculated by solving the optimization problem

$$
\begin{array}{ll}
\min & r_{i} \\
\text { s.t. } & B_{i} \geq B_{i+1}, \quad \sum_{i=1}^{N} B_{i}<B_{g} \\
& r_{i} \geq r_{\min } \\
& T_{y i} \leq T_{y(i+1)}, \quad \tau<T_{y i}, \quad \forall i=1,2, \ldots, N,
\end{array}
$$

where $r_{\min }$ denotes the minimum transmission rate that guarantees that no packet dropout occurs in the course of data transmission and $r_{i}$ denotes the actual transmission rate of the $i$ th output channel.

Meanwhile, different channel (input and output) is sampled with different sampling period making the dynamic bandwidth allocation possible since the synchronous data transmission is avoided in NMCSs. In time-driven manner, a subsystem with no data transmission currently can release its own bandwidth to others till the next transmission starts. These extra bandwidths can be assigned to other subsystems to accomplish data transmission faster. Such dynamic bandwidth allocation strategy, on one hand, reduces the network load and induced delays to some extent, on the other hand, guarantees the QoP of the all subsystems.

For example, in a MRO-NCS with two dependent subsystems, the outputs are sampled with periods $T_{y 1}, T_{y 2}$, respectively, and $T_{y 1}<T_{y 2}$. According to the aforementioned bandwidth allocation principle, the bandwidths assigned to the two subsystems are $B_{1}$ and $B_{2}$. Clear, $B_{1}>B_{2}$ and this guarantees sufficient transmission rate of both two channels. In a certain time interval $\Delta T$, by monitoring and evaluating the real-time performance of the whole system online, the sampling periods of the two channels are adjusted to $T_{y 1}^{\prime}$ and
$T_{y 2}^{\prime}$ since the outputs response is changed observably. Then, the bandwidths of the two subsystems are $B_{1}^{\prime}, B_{2}^{\prime}$ and $B_{1}^{\prime}>B_{2}^{\prime}$. The overall process can be illustrated in Figure 7 .

Generally speaking, the distinction of sampling period in NMCSs and the fast-slow and slow-fast characteristics of multirate sampling provide the possibility and methodology to achieve the dynamic bandwidth scheduling. The abovementioned control and scheduling codesign method can guarantee both the QoP of the control system and QoS of the communication network simultaneously by comprehensively considering the unique characteristics of multirate sampling.

\section{Conclusions and Future Works}

This survey paper has introduced and reviewed the fundamental issues and prospective directions for NMCSs. One can draw the conclusions that the introduction of multirate sampling in NCSs, on the one hand, will make the modeling, analysis, and synthesis of NCSs more complicated and challenge the achieved theoretical results,and, on the other hand, it will bring opportunities toward some higher goals for NCSs. Problems should be considered in the near future highlight as follows.

(1) Much effort has been directed to NCSs with variable sampling rate, but most results investigate the stability for a given worst-case interval between consecutive sampling times. This generally leads to conservative results that could be improved by taking into account a stochastic characterization for the intersampling times.

(2) A combination of multirate sampling and variable sampling in NCSs design may realize the allocation of network bandwidth and reduction of networkinduced delays preferably. Namely, multirate sampling implements on subsystems and variable sampling implements within each closed loop.

(3) Some other basic properties of control system such as controllability and observability should be reconsidered before they can be applied to networked setting.

From the point of view of the development of the control theory, multirate sampling complies with the trends of largescale, high distribution level and networked framework of computer control system and the research of NMCSs will be bound to further promote the development of the relevant theory and applications of NCSs.

\section{Conflict of Interests}

The authors declare that there is no conflict of interests regarding the publication of this paper.

\section{Acknowledgments}

This work was supported by the Natural Science Foundation (61263003), Fundamental Research Funds for the Central Universities (2009JC11 and 2009QN120) of China, 
and Fundamental Research Funds for Universities of Gansu Province. Also, it was partially supported by Doctor Foundation (BS03200901) and Excellent Young Teachers Foundation (Q201012) of Lanzhou University of Technology.

\section{References}

[1] Y. Tipsuwan and M.-Y. Chow, "Control methodologies in networked control systems," Control Engineering Practice, vol. 11, no. 10, pp. 1099-1111, 2003.

[2] T. C. Yang, "Networked control system: a brief survey," Institution of Electrical Engineers Proceedings: Control Theory and Applications, vol. 153, no. 4, pp. 403-412, 2006.

[3] J. P. Hespanha, P. Naghshtabrizi, and Y. Xu, "A survey of recent results in networked control systems," Proceedings of the IEEE, vol. 95, no. 1, pp. 138-172, 2007.

[4] J. Baillieul, "Special issue on networked control systems," IEEE Transactions on Automatic Control, vol. 49, no. 9, pp. 1421-1423, 2004.

[5] P. Antsaklis and J. Baillieul, "Special issue on technology of networked control systems," Proceedings of the IEEE, vol. 95, no. 1, pp. 5-8, 2007.

[6] D. P. Glasson, "Development and applications of multirate digital control," IEEE Control Systems Magazine, vol. 3, no. 4, pp. 2-8, 1983.

[7] Z. Xia, Z. Wei, Z. Hao, and C. Qijun, "Modeling of multirate input and output networked control system," in Proceedings of the Global Congress on Intelligent Systems (GCIS '09), pp. 258263, May 2009.

[8] X. Zhang and J. Xiao, "On the optimal multirate control of networked control systems," WSEAS Transactions on Systems, vol. 7, no. 7, pp. 721-731, 2008.

[9] Z.-W. Wang and G. Guo, "On model-based networked control system with multi-rate input sampling," International Journal of Modelling, Identification and Control, vol. 10, no. 1-2, pp. 160166,2010

[10] W. Chen and L. Qiu, "Stabilization of multirate networked control systems," in Proceedings of the 50th IEEE Conference on Decision and Control and European Control Conference (CDCECC '11), pp. 5274-5280, Orlando, Fla, USA, December 2011.

[11] Y. Zou, T. Chen, and S. Li, "Network-based predictive control of multirate systems," IET Control Theory and Applications, vol. 4, no. 7, pp. 1145-1156, 2010.

[12] Z. H. Guan, C. X. Yang, and J. Huang, "Stabilization of networked control systems with random delays: a new multirate method," in Proceedings of the 17th World Congress of the International Federation of Automatic Control, pp. 4204-4209, Seoul, Republic of Korea, 2008.

[13] Y. S. Suh, H. H. Lee, Y. S. Ro, and M. J. Yi, "Networked control systems using $\mathrm{H}_{2}$ multirate control," in Proceedings of the IEEE International Workshop on Factory Communication Systems (WFCS '04), pp. 403-406, Vienna, Austria, September 2004.

[14] Z. Li and M.-Y. Chow, "Adaptive multiple sampling rate scheduling of real-time networked supervisory control systempart I," in Proceedings of the 32nd Annual Conference on IEEE Industrial Electronics (IECON '06), pp. 4604-4609, November 2006.

[15] Z. Li and M.-Y. Chow, "Adaptive multiple sampling rate scheduling of real-time networked supervisory control systempart II," in Proceedings of the 32nd Annual Conference on IEEE Industrial Electronics (IECON '06), pp. 4610-4615, November 2006.
[16] R. E. Kalman and J. E. Bertram, "A unified approach to the theory of sampling systems," Journal of the Franklin Institute, vol. 267, no. 5, pp. 405-436, 1959.

[17] A. Ray and Y. Halevi, "Integrated communication and control systems-part II: design considerations," Journal of Dynamic Systems, Measurement and Control, Transactions of the ASME, vol. 110, no. 4, pp. 374-381, 1988.

[18] L. Dritsas, G. Nikolakopoulos, and A. Tzes, "On the modeling of networked controlled systems," in Proceedings of the Mediterranean Conference on Control and Automation (MED '07), Athens, Greece, July 2007.

[19] Z. Wang, Z. Li, and D. Luo, "Modeling and control of networked systems with different loop topologies," in Proceedings of the International Conference on Advanced in Control Engineering and Information Science (CEIS '11), pp. 594-600, August 2011.

[20] J. Xiao, Multi-Rare Digital Control Systems, Science Publishing House, Beijing, China, 2003.

[21] Q. Zhu and G. Xie, "Analysis and modeling of multi-rate networked control systems with long time delay," in Proceedings of the 24th Chinese Control and Decision Conference (CCDC '12), pp. 2978-2983, 2012.

[22] G. Kreisselmeier, "On sampling without loss of observability/ controllability," IEEE Transactions on Automatic Control, vol. 44, no. 5, pp. 1021-1025, 1999.

[23] G. Guo, "Systems with nonequidistant sampling: controllable? Observable? Stable?" Asian Journal of Control, vol. 7, no. 4, pp. 455-461, 2005.

[24] L. F. Godbout Jr., D. Jordan, and M. E. Striefler, "Pole placement algorithms for multirate-sampled linear systems," Automatica, vol. 30, no. 4, pp. 723-727, 1994.

[25] P. Colaneri, R. Scattolini, and N. Schiavoni, "Stabilization of multirate sampled-data linear systems," Automatica, vol. 26, no. 2, pp. 377-380, 1990.

[26] P. G. Voulgaris, M. A. Dahleh, and L. S. Valavani, " $H_{\infty}$ and $\mathrm{H}_{2}$ optimal controllers for periodic and multirate systems," Automatica, vol. 30, no. 2, pp. 251-263, 1994.

[27] P. G. Voulgaris and B. Bamieh, "Optimal $H_{\infty}$ and $H_{2}$ control of hybrid multirate systems," Systems and Control Letters, vol. 20, no. 4, pp. 249-261, 1993.

[28] P. P. Khargonekar, K. Poolla, and A. Tannenbaum, "Robust control of linear time invariant plants using periodic compensation," IEEE Transactions on Automatic Control, vol. 30, no. 11, pp. 1088-1096, 1985.

[29] M. E. Sezer and D. D. Siljak, "Decentralized multirate control," IEEE Transactions on Automatic Control, vol. 35, no. 1, pp. 6065, 1990.

[30] P. T. Kabamba and Y. Chang, "Simultaneous controller design for linear time-invariant systems," IEEE Transactions on Automatic Control, vol. 36, no. 1, pp. 106-111, 1991.

[31] A. Locatelli and N. Schiavoni, "Simultaneous regulation of multirate sampled-data systems," in Proceedings of the American Control Conference, pp. 1503-1507, Seattle, Wash, USA, June 1995.

[32] J. Xiao and L. Tang, "Pole assignment of multirate digital control systems with decentralized structure," in Proceedings of the International Workshop on Autonomous Decentralized System, pp. 216-219, 2000.

[33] C. L. Liu and J. W. Layland, "Scheduling algorithms for multiprogramming in a hard real-time environment," The Journal of the Association for Computing Machinery, vol. 20, no. 1, pp. 4661, 1973.

[34] M. S. Branicky, S. M. Phillips, and W. Zhang, "Scheduling and feedback co-design for networked control systems," in 
Proceedings of the 41st IEEE Conference on Decision and Control, pp. 1211-1217, December 2002.

[35] F.-L. Lian, J. Moyne, and D. Tilbury, "Network design consideration for distributed control systems," IEEE Transactions on Control Systems Technology, vol. 10, no. 2, pp. 297-307, 2002.

[36] J. Q. He and H. C. Zhang, "Optimal sampling period selection method for network control system," Journal of JiLin University, vol. 34, no. 3, pp. 479-482, 2004.

[37] J.-Q. He, H.-C. Zhang, and Y.-Z. Jing, "Study on feedback scheduling for NCS with packet losses," Journal of the University of Electronic Science and Technology of China, vol. 35, no. 5, pp. 791-822, 2006.

[38] S. H. Hong, "Scheduling algorithm of data sampling times in the integrated communication and control systems," IEEE Transactions on Control System Technology, vol. 3, pp. 225-231, 1995.

[39] H. S. Park, Y. H. Kim, S. Kimd, and W. H. Kwon, "A scheduling method for network based control systems," IEEE Transactions on Control System Technology, vol. 10, pp. 318-330, 2002.

[40] S. H. Hong, "Scheduling algorithm of data sampling times in the integrated communication and control systems," IEEE Transactions on Control Systems Technology, vol. 3, no. 2, pp. 225-230, 1995.

[41] D. Yue, Q.-L. Han, and C. Peng, "State feedback controller design of networked control systems," IEEE Transactions on Circuits and Systems II: Express Briefs, vol. 51, no. 11, pp. 640644, 2004.

[42] K. Peng, X.-F. Li, L. Chen, and J.-D. Chen, "Determination of optimal sampling frequencies in multi-loop networked control system," Control and Decision, vol. 19, no. 10, pp. 1151-1154, 2004.

[43] C. Peng, D. Yue, Z. Gu, and F. Xia, "Sampling period scheduling of networked control systems with multiple-control loops," Mathematics and Computers in Simulation, vol. 79, no. 5, pp. 1502-1511, 2009.

[44] C. Peng, D. Yue, and Z. Gu, "Multiple sampling periods scheduling of networked control systems," in Proceedings of the 27th Chinese Control Conference (CCC '08), pp. 447-451, Yunnan, China, July 2008.

[45] W. Yan and J. Zhicheng, "Switched feedback control for wireless networked control system with adaptive sampling rate," in Proceedings of the 31st Chinese Control Conference, July 2012.

[46] Z. W. Wang and H. T. Sun, "Bandwidth scheduling of networked control system based on time varying sampling period," in Proceedings of the 32nd Chinese Control Conference, July 2013.

[47] Z. W. Wang and H. T. Sun, "Bandwidth allocation strategy of networked control system based on multirate sampling method," International Journal of Digital Content Technology and Its Applications, vol. 6, no. 23, pp. 651-659, 2012.

[48] Z. W. Wang and S. H. Han, "Dynamic scheduling of networked control systems based on variable-sampling," Submit to the 25th Chinese Process Control Conference.

[49] D. Steo, J. Lehoczky, P. L. Sha, and K. G. Shin, "On task schedulability in real-time control systems," in Proceeding of the IEEE Conference on Decision and Control, vol. 4, pp. 865-870, Piscataway, NJ, USA, 2000. 


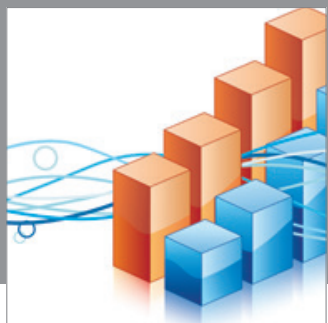

Advances in

Operations Research

mansans

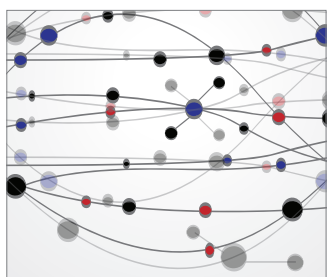

The Scientific World Journal
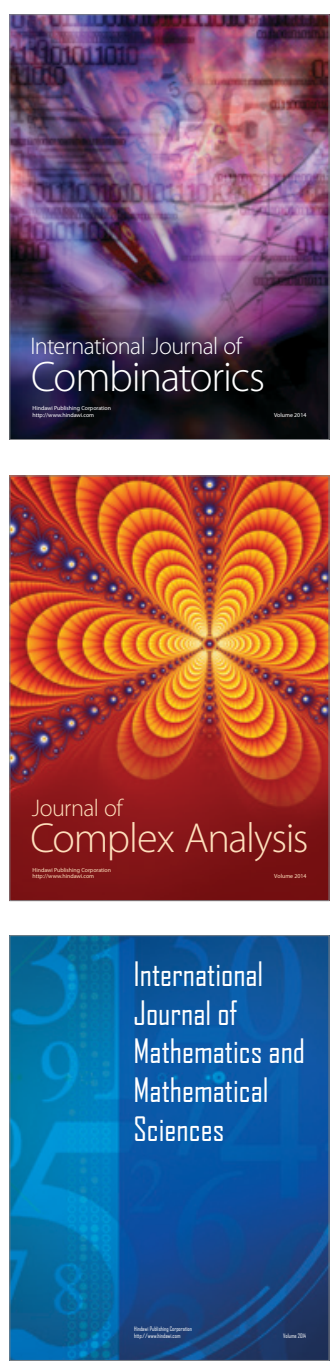
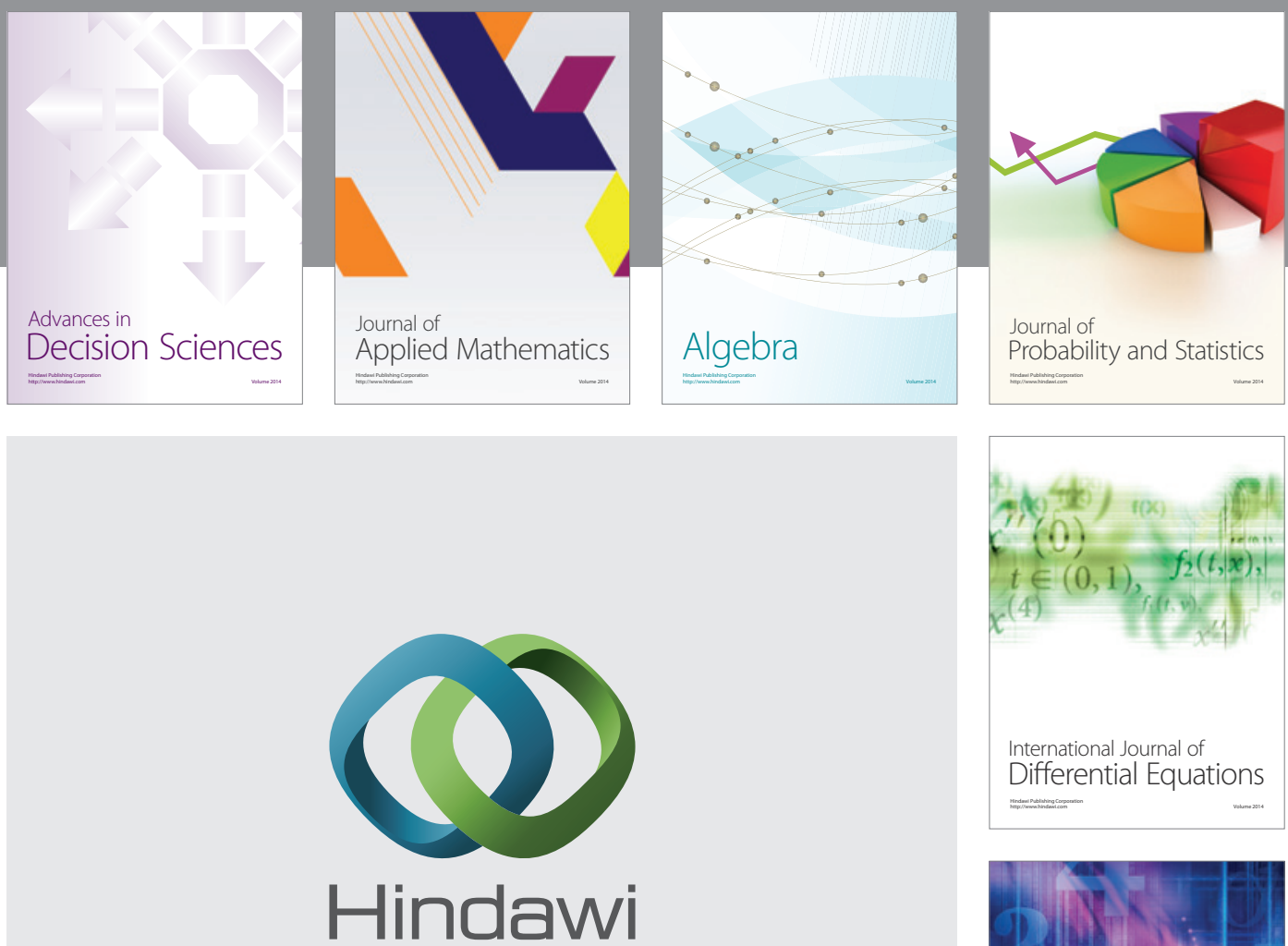

Submit your manuscripts at http://www.hindawi.com
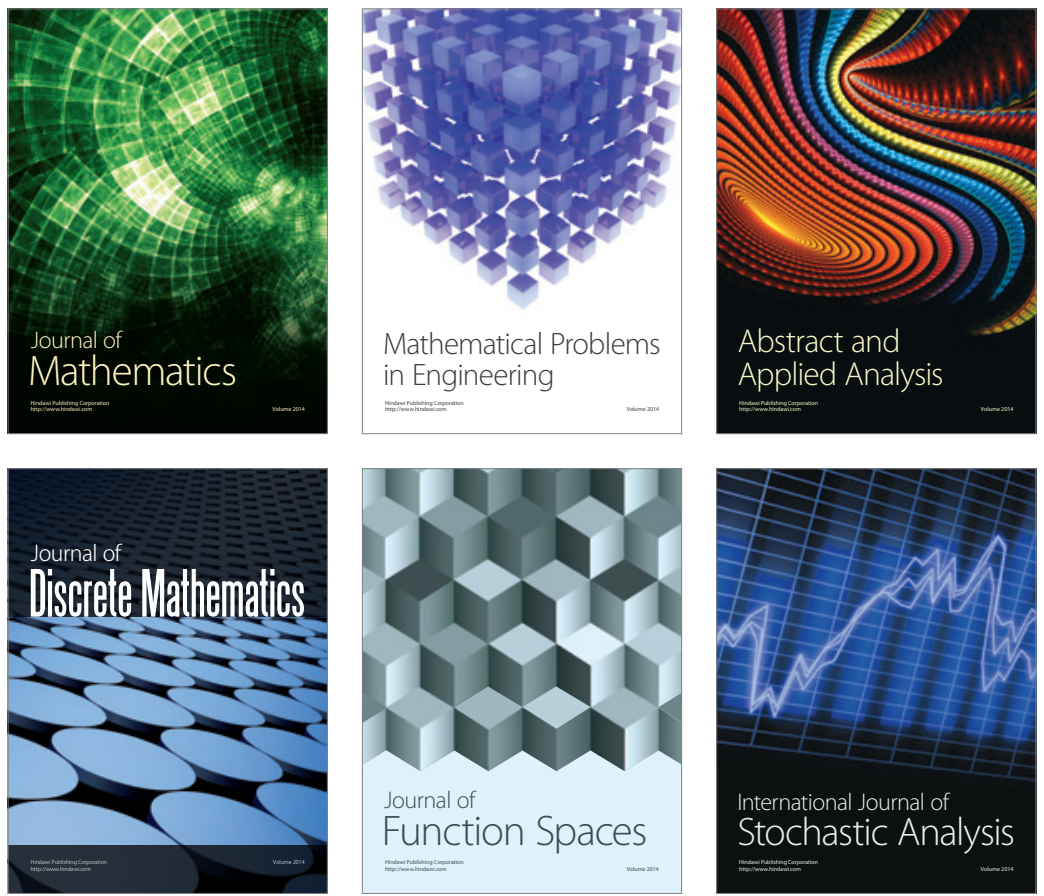

Journal of

Function Spaces

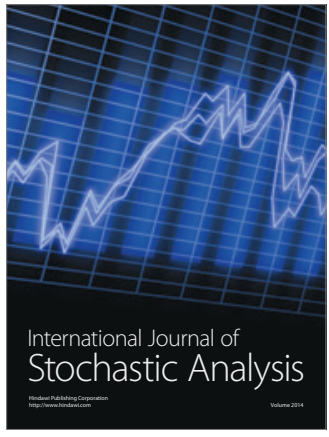

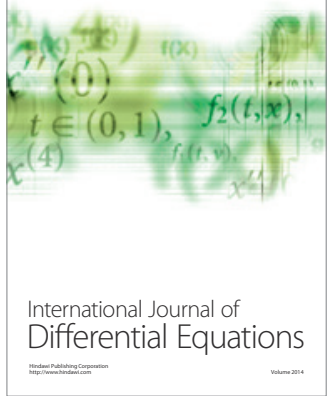
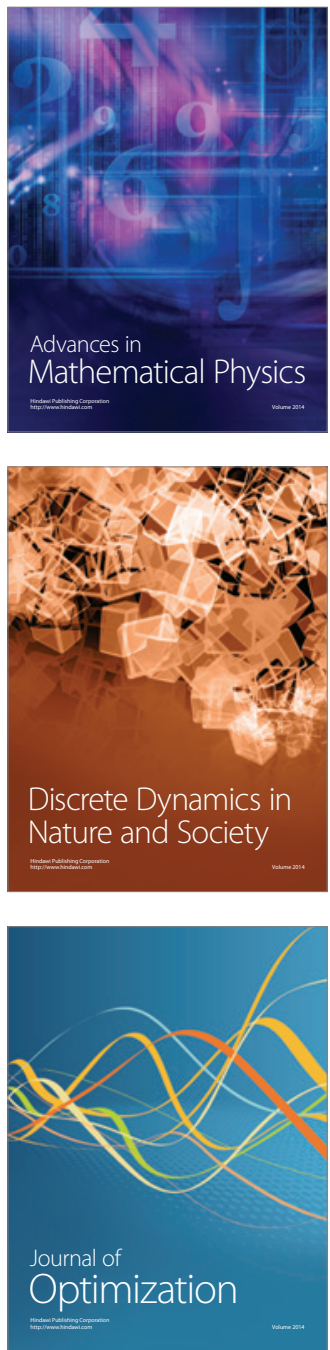